\title{
COMBATING THE 'SICK BUILDING SYNDROME' BY IMPROVING INDOOR AIR QUALITY
}

Pongchai Nimcharoenwon and Graham Miller, School of Construction, Property And Planning, University of Western Sydney, New South Wales, Australia

\section{Introduction}

\subsection{Sick Building Syndrome (SBS)}

The Sick Building Syndrome (SBS) is a term often used to explain symptoms, such as headaches, dizziness, runny noses, itchiness and so on, produced by any harmful environment in buildings, especially in air-conditioned buildings (Royal Australian Institute of Architects, 1991, p.1). As approximately 80 to $90 \%$ of people's time is spent in buildings (EAP, 1998,), concerns about indoor air quality resulting in such symptoms are increasing.

There are a number of methods which appear to be able to reduce the SBS symptoms, such as purifying/cleaning indoor air and utilising materials for construction, furniture and finishes that have been proven to reduce adverse effects. O'Brian (1988) and Karnstedt (1991), suggest that ionised air is beneficial to human health and can also reduce the symptoms of SBS.

Based on the premise that, if SBS and the potential for harmful conditions within buildings are to be avoided, building professionals require suitable design tools (Ferguson 1987, p.1), this paper describes a method for estimating how much additional negative ions should be added to a room/office to compensate for the losses caused by three major factors namely, video display terminals (VDT), heating ventilation and air-conditioning (HVAC) and building contents (BC).

\subsection{Negative lons}

Ions are electrically charged atoms or molecules that can gain or lose an electron (Bionic Products, 1998). An atom gaining electron(s) is called a negative ion and an atom losing electron(s) are called a positive ion. From this formation process of the ions, there seem to be a number of ion effects, positively and negatively in terms of indoor air quality. With regard to the nature of the movement of electrons, negative ions (atoms with excess electrons) are quicker than positive ions. The positive effects of this phenomenon can be felt near waterfalls, in pine forests and on the seashore where waves are breaking on the shore (Bionic Products, 1998). Most negative ions are likely to be the negative hydrogen ions $(-\mathrm{H})$ produced by solar continuous spectrum (Massey, 1976, p.673). Massey suggests that the number of negative ions of carbon $(-C)$ and oxygen $(-O)$ produced by ozone reaction are significant. These types of negative ions are therefore also considered for the purposes of the calculations used in this study.

\subsection{Negative Ion Therapy}

There has been a considerable amount of research that has shown positive and beneficial effects from negative ions such as removing air particles and contaminants, and preventing and reducing the SBS (O'Brian, 1988, and Karnstedt, 1991). Some researchers however, are sceptical about the positive effects. They suggest that the reverse may be true and that generating negative ions might emit ozone excess that can irritate eyes and affect the lungs (Nava, 1998). It is not the aim of this study to argue either way but rather to demonstrate a method for estimating the de-ionising effects of key factors in modern buildings.

The following tables show the possible effects of the amount of negative ions in particular areas (Penex Air lonisers, 1988). 
Table 1.1: Possible Effects of Negative Ion Density

\begin{tabular}{|l|l|}
\hline Density (ions/cc) & Effects \\
\hline $0-100$ & $\begin{array}{l}\text { Dead air, oppressive, difficulty of concentration; virus's and } \\
\text { germs flourish. }\end{array}$ \\
\hline $500-1000$ & $\begin{array}{l}\text { Normal air found indoors where pollution is low and the building } \\
\text { has open windows. }\end{array}$ \\
\hline $1000-5000$ & $\begin{array}{l}\text { Country fresh air, the minimum level one should sleep, work } \\
\text { and live in. }\end{array}$ \\
\hline $5000+$ & \begin{tabular}{l} 
Exceptionally fresh, clean and invigorating air, "mountain air." \\
\hline $50,000+$
\end{tabular} \\
\hline
\end{tabular}

Table 1.2: Negative Ion Densities in Places or Conditions

\begin{tabular}{|l|l|}
\hline Density (ions/cc) & Places or Conditions \\
\hline $0-250$ & $\begin{array}{l}\text { Hermetically sealed steel-structure office building, with central } \\
\text { heating/Air conditioning }\end{array}$ \\
\hline $20-251$ & Inside and Airplane \\
\hline $0-100$ & Smoky indoor air \\
\hline $250-500$ & Normal indoor air (windows open) \\
\hline $250-750$ & Urban Air in average industrial city \\
\hline $1,000-2,000$ & Country Air \\
\hline $1,000-5,000$ & Mountain Air \\
\hline $5,000-20,000$ & Inside Caves \\
\hline $25,000-100,000$ & Waterfalls \\
\hline
\end{tabular}

\section{NEGATIVE ION LOSS IN AIR- CONDITIONED BUILDINGS}

\subsection{Factors Decreasing Negative lons}

Negative ions in air-conditioned buildings may be reduced by a number of means. In air-conditioned offices principal reducers of negative ions are, Video Display Terminals (VDT's), HVAC (particularly airconditioning systems), and Building Contents (BC's) including building materials, furniture and finishes used. Figure 1 shows the effects of the three factors reducing negative ions.

As can be seen from the figure 1, each factor reduces the amount of negative ions in buildings differently. VDT's, appears to decrease negative ions by emitting ionising radiation as low energy $x$ ray. HVAC is likely to reduce the negative ion density (ions/cc) in air through its process, especially the process of the airconditioning system. The Building Contents (BC's), which naturally have electrostatics, may be electrically activated by contact, such as sliding and rolling of two insulating materials. The electrical charges generated appear to attract the positive and negative ions in indoor air and stick on the BC's surfaces, and therefore the ions in buildings are likely to be reduced. 


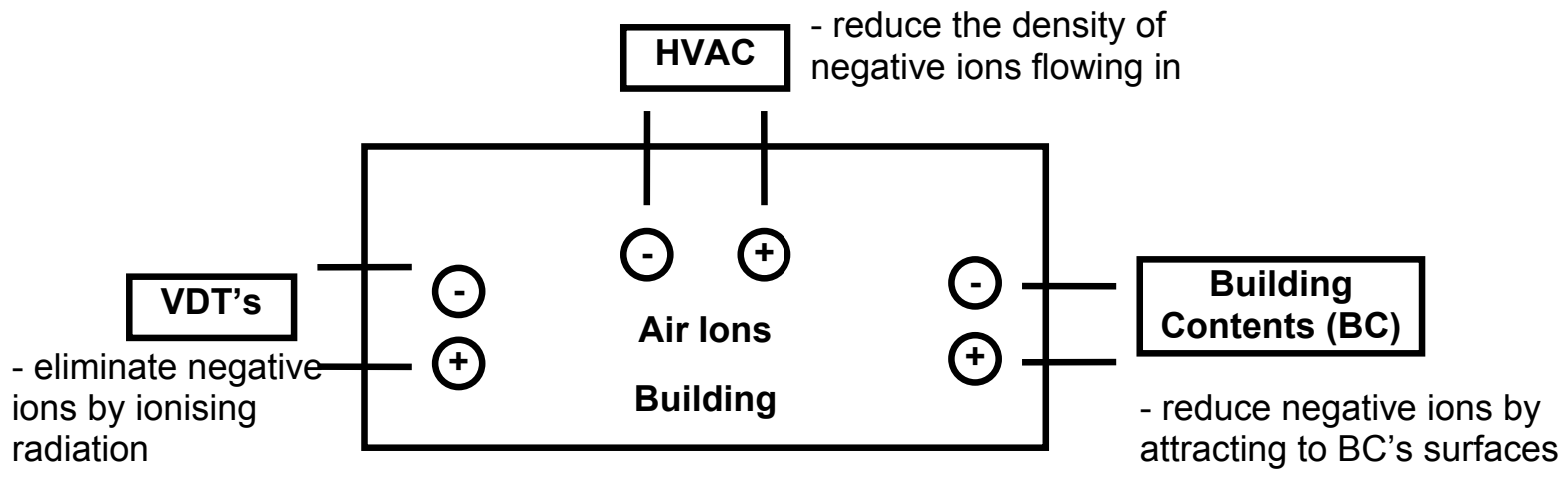

Figure 1: Three main factors affecting negative ion decrease in buildings

2.2 How much Each Factor Reduces Negative lons

\subsubsection{Video Display Terminals (VDT’s)}

Studies of VDT's by the Australian Radiation Laboratory, (1998), indicate that the while terminals emit visible radiation, in creating the image, many types of the electromagnetic radiation are also generated, in particular, Extremely Low
Frequency radiation (ELF), and low energy $x$-ray. The latter is an ionising radiation, which can knock electrons out of negative ions by overcoming the electron binding energy or electron affinity (EBE) of negative ions (Australian Radiation Laboratory, 1998, lonising Radiation Quantities And Unit). The negative ions then lose their potential (see also figure 2).

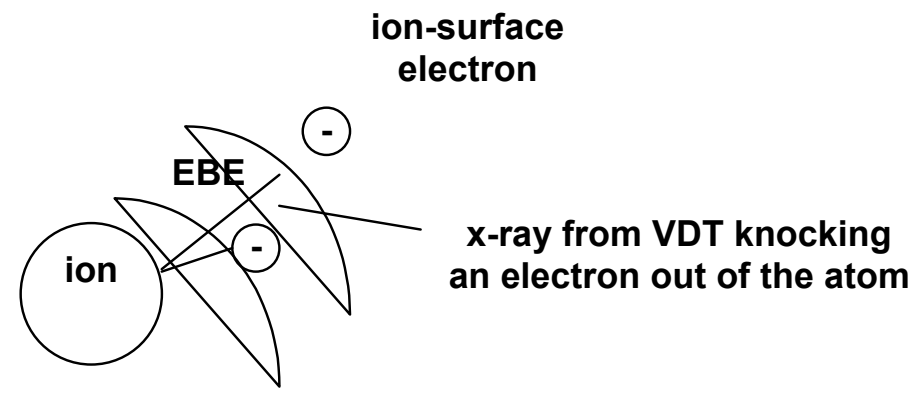

Figure 2: the typical positions of electrons in an ion

As a consequence of this, the energy of low energy $x$-ray and the electron binding energy or electron affinity of negative ions (EBE) can be calculated and consequently the amount of negative ions eliminated can also be calculated.

The following equation indicates the relationship of participant parameters for estimating Negative lon Loss from VDT's (VDT-NIL).
VDT-NIL $\alpha$ LEX, 1/EBE, Rr, and Fn (Equation 1)

Where:

LEX = Low Energy X-ray = approx. 9 to 20 keV per a VDT (ARL, 1998, Calibration: Therapy Dosimeter Radiation Qualities) $\mathrm{EBE}=$ Electron Binding Energy of $-\mathrm{H}$ and $\mathrm{O} 2$ = approx. 0.277 and $0.440 \mathrm{eV}$ (Massey H., 1976, p.9 and 184) 
$\mathrm{Rr}=$ Refresh rate of VDT's $=$ approx.50 to $95 \mathrm{~Hz}$ (Tijierina L., 1984, p.7)

$\mathrm{Fn}=$ Negative lon Factor $=$ approx.4/9 (ration between ordinarily positive and negative ions)

From the relationship in Equation (1), the Negative lon Loss (NIL) by VDT's can be estimated in terms of the negative ions reduced in a period of operating time as follows:

VDT-NIL $=4.7 \times \mathrm{Nv} \times \mathrm{PO}+-15 \%$ bil. ions (Equation 2)

Where:

$\mathrm{NV}=$ Number of the VDT's

$\mathrm{PO}=$ Period of Operating the VDT's

This above Equation (2) shows the loss of negative ions by the low energy $x$-ray of a VDT. Moreover, it determines by referring to the possible effect of the loss, to the lowest electron binding energy of negative ions. As an example two normal 14" or 15" television and computer screens might eliminate almost all the negative ions (typically 500 neg.ions/cu.cm) in a closed room of $3 \times 3 \times 2.4 \mathrm{~m}$ within 30 mins.
The Negative Ion Loss from HVAC (HVAC-NIL) can be calculated from the fact that particles in air are likely to be electrically charged by an electric field intensity (about $30 \mathrm{kV} / \mathrm{cm}$ ) in any normal condition including air-conditioned buildings (Hendricks, cited in Moore, 1973, p.57). They then attract the opposite charge of ionised air (negative or positive charge), and thus a number of negative ions will stick their electrons on the particle surfaces (see also figure 4). Each negative ion is assumed to have an excess electron of approximate $1.6 \times 10^{\wedge}$ 19 C (Weisstein, 1996)

After the air conditioning system filters a number of the atmospheric dust and/or particles, a number of negative ions seem to be also obstructed by filtering. The process is to consider filtering atmospheric particles, and assume the particles as spheres. The electric field intensity can be considered as an average value, which includes the intensity of the air conditioning system and others (Hendricks, cited in Moore, 1973, p.57)

\subsubsection{HVAC}

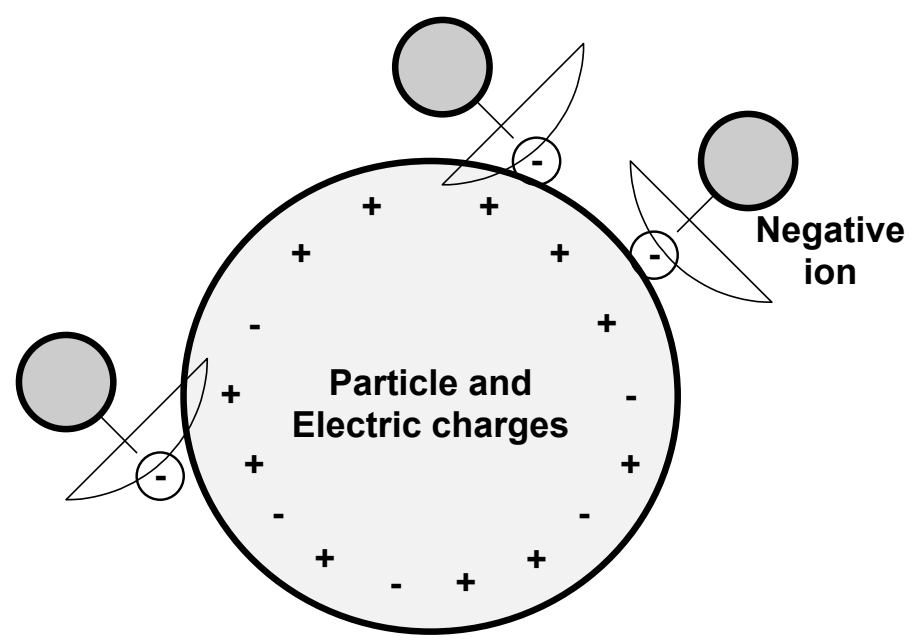

Figure 4: Cross section showing negative ions attracting a particle

The Negative lon Loss (HVAC-NIL) is estimated as a percentage of the difference between negative ions outside and inside (\%NIL). The following equation shows the relationship of the participant parameters for this.
$\%$ NIL $\alpha$ Qp, rp, Fn, Dneg, 1/Cp, Fh, \%Ef, and $1 / \mathrm{Dp} \quad$ (Equation 3)

Where:

$\mathrm{Qp}=$ Charge of a particle $=3.1 \times 10^{\wedge}-4 \times$ $\mathrm{R}^{\wedge} 2$ (Hendricks, cited in Moore, 1973, p.59) 
$\mathrm{rp}=$ Radius of a particle

$\mathrm{Fn}=$ Negative Ion Factor $=$ approx.4/9

(ration between ordinarily positive and negative ions)

Dneg $=$ Density of negative ions outside (ions/cc)

$\mathrm{Cp}=$ Particle concentration (gm/cu.m)

$\mathrm{Fh}=\mathrm{HVAC}$ flow rate $(\mathrm{cc} / \mathrm{s})$

$\% E f=$ Filtration efficiency of HVAC (air conditioning systems)

$\mathrm{Dp}=$ Density of particles (particles/gm)

From the relationship (equation 3), the percentage of negative ions reduced $(\% \mathrm{NIL})$ in any area type (or in any particle concentration) can evaluated as shown in figure 5. The parameters assumed are that the flow rate of the air conditioning system (Fh), and negative ions density outside (Dneg) are 200 CFM and 200 to 1000 ions/cc, respectively. For example, in urban air the density (ions/cc) of indoor negative ions is approximately 150 ions/cc.

\subsubsection{Building Contents (BC)}

This section illustrates the effects of electrostatics on the BC's surfaces such as building materials and furniture. The effect seems to come from normal activities of the occupants in buildings, such as sitting on a chair, documents being put on a table, walking on a floor, and so on (Electro Statics, 1998). Such activities may potentially bring about electrically charged surfaces. The electrically charged ions (negative and positive ions) in the indoor air will be attracted (polarised) by the charged surfaces and eventually be reduced in the air.

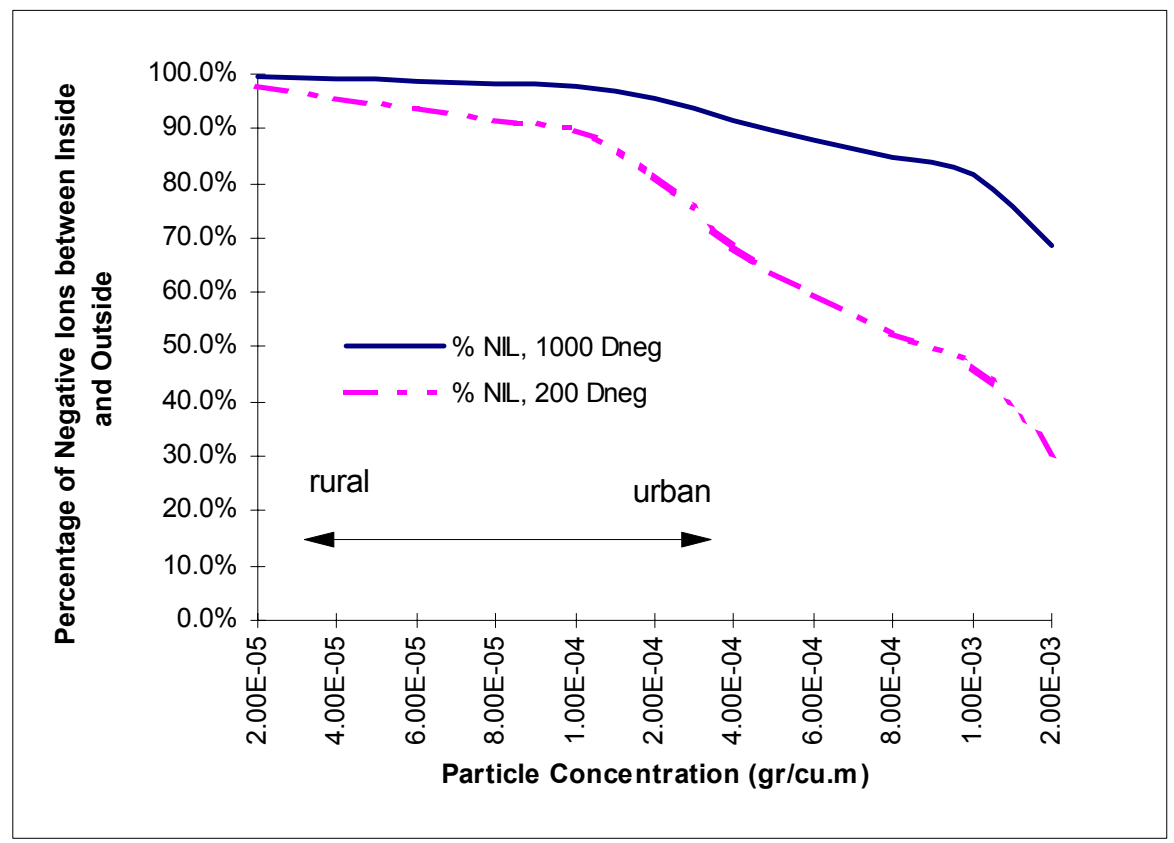

Figure 5: Relationship between \%NIL and Particle Concentrations

'When two insulating materials are rubbed together, the surfaces acquire a net electric charge, with one becoming negative and the other positive.' (Cross, 1987, p.17). This is the action of the electrostatic potential on the material surfaces. In addition, the action is likely to be caused by contact between two materials. The unbalancing charges, after that, occur at the material surfaces and become polarising. Effectively, air ions will be attracted by the opposite charged surface and give up their potential (Jowett, 1976, p.56). Figure 6 shows the simply contacting action of two materials during and after contacting. 


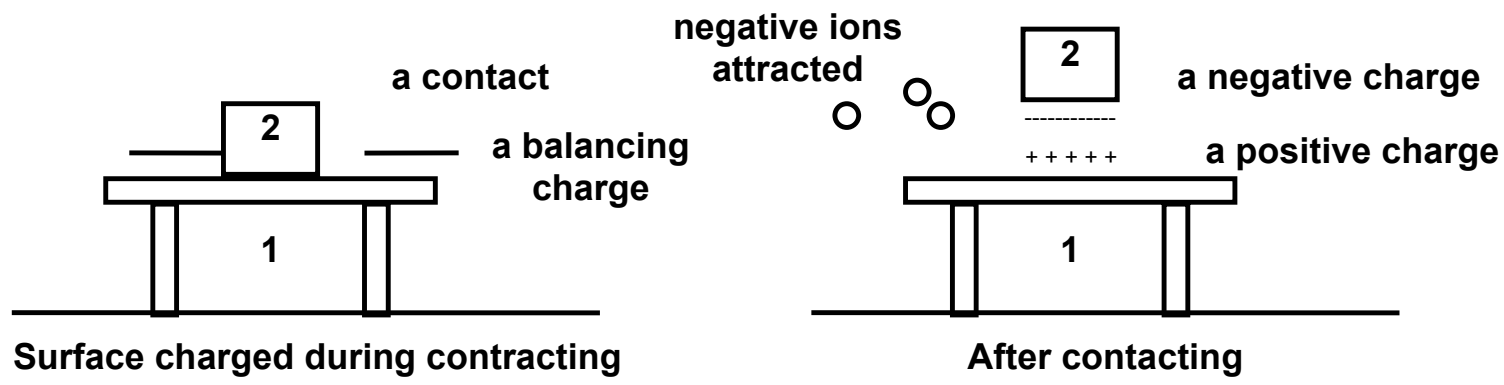

Figure 6: Electric charge surfaces of two building materials

From the effective contacting of two insulators (see also figure 6), the relationship between the negative ions attracted by the positive charges and related parameters can be demonstrated as follows:

BC-NIL $\alpha \sigma, \% \sigma$,max., and $1 / \mathrm{R} \sigma, \mathrm{m} / \mathrm{i}$ (Equation 4)

Where:

$\sigma=$ ideal surface charge density of two materials $=$ approx.1.656 $\times 10^{\wedge} 10 \mathrm{e} / \mathrm{sq} . \mathrm{cm}$ (Jowett, 1976, p.116)

$\% \sigma$, max $=$ the maximum percentage of sliding and rolling contacts = approx. $8 \%$ (Jowett, 1976, p.116)

$\mathrm{R} \sigma, \mathrm{m} / \mathrm{i}=$ Ratio between the surface charge density of metal and insulate materials $=$ approx $\cdot 10,000$ to 26,000

As a consequence of this, while the material surfaces are electrostatically activated and become electrical charge, negative and positive ions seem to be attracted by polarisation of the opposite surface charges of about $5.095 \times 10^{\wedge} 4$ to $1.325 \times 10^{\wedge} 5$ e/sq.cm. Equation (5) is a formula to estimating Negative Ion Loss from the Building Contents (BC).

BC-NIL $=\max .1 .325 \times 10^{\wedge} 9 \times$ NoCT $x$ Abc ions (Equation 5)

Where:

NoCT $=$ Number of effective Contacts $A b c=$ surface Area of Building Contents contacted
An example of this is in an office building where the contents, the seat(s) of chairs used, computer keyboard(s) used and so on, may affect the Static Electrification. If we assume their areas to be about 5 sq.m, this room will lose approximately a maximum of 6.63 bil.ions of negative ions.

\section{NEGATIVE ION LOSS TESTING}

\subsection{DOF-NIL Formula}

Three Negative lon Loss formulas can be incorporated and become DOF-NIL formula. The relationship between the DOF-NIL and participant parameters is demonstrated in the following equation. DOF-NIL a Nv, PO, AT, FR, NoCT, and Abc (Equation 6)

Where:

Nv = Number of VDT's

$\mathrm{PO}=$ Period of Operating time of VDT and HVAC

AT = Area Type (urban or rural)

$\mathrm{FR}=$ air Filtration Rate of HVAC (airconditioning system)

$\mathrm{FE}=$ Filtration Efficiency of HVAC (airconditioning system)

NoCT $=$ Number of effective Contacts of two insulate materials

Abc $=$ surface Area of a Building Content contacted

3.2 Testing Negative Ion Loss In Airconditioned Offices

To validate the results provided by the formula, testing of Negative Ion Loss (NIL) was performed using an instrument called an Air lon Counter (AIC). The test was 
performed in two different areas: a typical air-conditioned office $(3 \mathrm{~m} \times 3 \mathrm{~m} \times 2.4 \mathrm{mH})$, and a room $(6 \mathrm{~m} \times 3 \mathrm{~m} \times 2.4 \mathrm{mH})$, which was used for testing only the effects of an air conditioning system, and the density of negative ions outside the rooms (Dneg) about 1,500 ions/cc.

DOF-NIL showed the results in terms of negative ion density (ions/cc) in the rooms, and the rate (ions/hr) of negative ions reduced by VDT's and BC's with +$15 \%$ error of VDT and BC effects. The AIC showed the negative-ion density (ions/cc) in each room with $25 \%$ accuracy.

The results were as follows:

In the air-conditioned office and the control room, the negative ion densities inside were about 1,400 and 1,450 ions/cc with the negative ion decrease

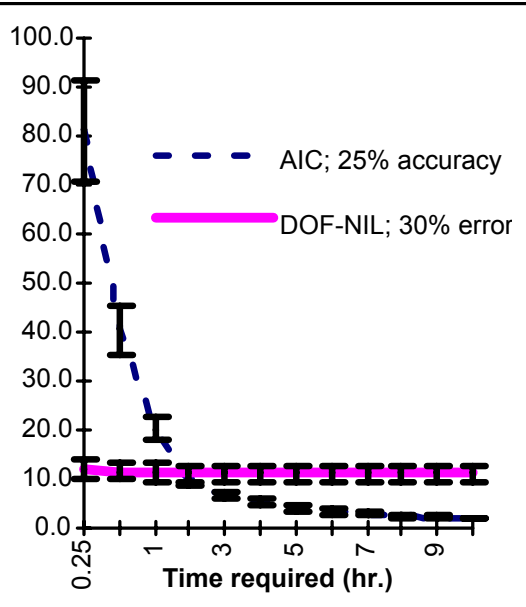

Figure 7.1: Typical Office rates of about $1 \times 10^{\wedge} 10$ and $1 \times 10^{\wedge} 9$ ions $/ \mathrm{hr}$, whereas the $\mathrm{AIC}$ showed the density of about 600 and 1,250 ions/cc, respectively.

Figure 7 below, shows the rates of additional negative-ions required for the condition of 1,500 ions/cc in the required period of 1 to 10 hours. The additional rate indicated by the AIC drops sharply when longer ion-generation is required.

Whereas according to the DOF-NIL formula the rate remains quite stable over any time period. However the estimated required rate of additional negative-ions for a 3 to 4 hour period (a realistic time period) as calculated using the DOF-NIL formula compared favourably with results obtained by the Air lon Counter (AIC) - for an air-conditioned office this is approximately 12 billion ions $/ \mathrm{hr}$.

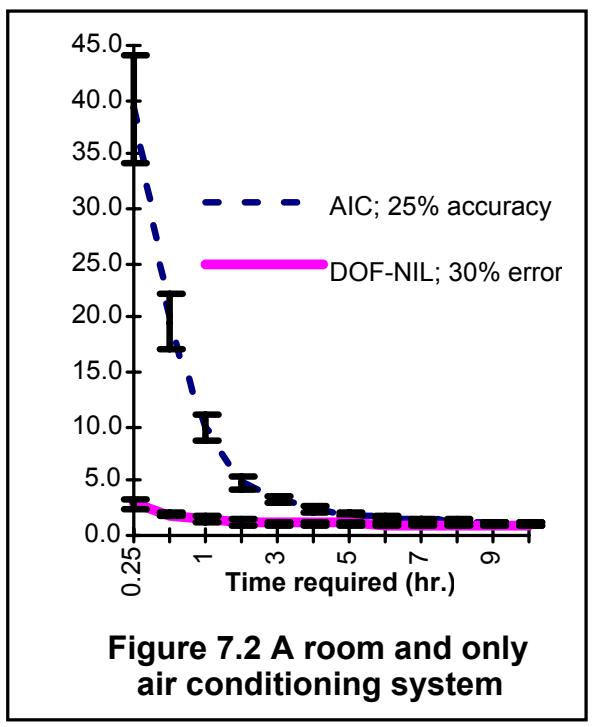

Figure 7: Additional Negative-lons for 1 to 10 hours required

\section{CONCLUSIONS}

It is proposed that the DOF-NIL formula described provides a method that has a reasonable degree of accuracy for estimating the amount of negative ion loss in air-conditioned buildings for the critical 3 to 4 hour period. The DOF-NIL formula can be used to estimate the loss in terms of negative ion density (ions/cc) by HVAC, and the rate (ions/hr) of negative ions reduced by VDT's and BC's. A weakness with the DOF-NIL formula, is that only considers three factors, whereas a suitable instrument can take account of significantly more factors, for example the effects of air leakages in buildings and the effect of particle size and density. However these factors and the additional factors measured by an instrument appear 
to be relatively minor in their effect, and the difference in the results obtained are therefore also minor. Currently available instruments require a considerable amount of time to record data - typically up to 10 hours and require a real building to obtain results, whereas applying the formula method can produce data quickly and easily which can be used at the design stage to avoid possibly costly mistakes and/or to introduce measures to counter the adverse effects of deionisation.

\section{REFERENCES}

Australian Radiation Laboratory ARL (1998), Calibration: Therapy Dosemeter Radiation Qualities, http:// www.health.gov.au:80/arl/irs_cal2.htm

Australian Radiation Laboratory ARL (1998), lonising Radiation Quantities And Unit, http://www. health.gov.au:80/arl/irs_bg2_htm (10 September, 1998)

Australian Radiation Laboratory ARL (1998), Radiation Emissions From Video Display Terminals,

http://www.health.gov.au:80/arl/is_vdtrd.ht m (10 September, 1998)

Bionic Products (1998), What Are lons, http://www.negativeions.com/Main.htm (10 October, 1998)

Cross, J.A. (1987), Electrostatics: Principles, Problems And Applications, Adam Hilger: Bristol

Electro Statics (1998), Static Electricity, http://www.electrostatics.com/page2.html (15 October, 1998)

Environmantal Protection Agency (1998), EPA's Indoor Air Quality Home Page, http://www.epa.gov/iaq (23 August, 1998)

Ferguson, D. (1987), Indoor Air Pollution: The Concern Of Architects dissertation 2, RAIA Practice Division: N.S.W.
Jowett, C.E. (1976), Electrostatics In The Electronics Environment, Macmillan Press: London

Karnstedt, J (1991, cited in Electrocorp, 1998), Ions And Consciousness, http:// www.net-gain.com/ electrocorp/cons.html (23 August, 1998)

Massay, H.S. (1976), Negative lons, Cambridge University Press: London

Mechanical Engineering Services (1992), Air Filters No.DA15: Application Manual, Australian Institute Of Refrigeration, Air Conditioning And Heating Inc.

Moore, A.D. (Ed.) (1973), Electrostatics and its Applications, John Wiley\& Sons, New York.

Nava G. (1998), No Positive Effects From Negative lons Therapy, http://www.netasia.net/users/truehealth /negative\%20ion\%20therapy.htm (24 September, 1998)

Penex Air lonisers (1998), What Are Negative lons, http://www.pentex.com/homepage. html (9 September, 1998)

O'Brien, R. cited in Electrocorp (1998), lons Can Do Strange Things To You, http://www.netgain.com/electrocorp/strange.html (23 August, 1998)

Royal Australian Institute of Architects (May, 1991), The Sick Building Syndrome: The Liability Of Architects In The Design Of Buildings report, RAIA Practice Division: N.S.W.

Tijerina, L. (1984), Video Display Terminals: Workstation Ergonomics, Online Computer Library Center Inc.: Columbus.

Weisstein, E.W. (1996-1998), Physics, http://www.astro.virginia.edu/eww6n/physics (22nd September, 1998). 